\title{
Twistors and the asymptotic behaviour of massless spin- $\frac{3}{2}$ fields
}

\author{
Jörg Frauendiener†, Jaideep Ghosh $\ddagger$ and Ezra T Newman $\ddagger$ \\ $\dagger$ Max-Planck-Institut für Gravitationsphysik, Albert-Einstein-Institut, Schlaatzweg 1, D-14473 \\ Potsdam, Germany \\ $\ddagger$ Department of Physics and Astronomy, University of Pittsburgh, Pittsburgh, PA 15260, USA
}

Received 27 October 1995, in final form 4 January 1996

\begin{abstract}
In this work the asymptotic solutions of massless spin- $\frac{3}{2}$ fields are studied. First, in a Minkowski space, the fields, their potentials, and the associated gauges are analysed in the Rarita-Schwinger description. We exhibit the explicit appearance of the conserved charges of the fields and their singular behaviour in the potentials. The charges of the spin- $\frac{3}{2}$ fields emerge as a global definition of twistors. The flat space case serves as a guide in the analysis of the problem in general vacuum spacetimes. Although the fundamental connection between and the meaning of twistors and charges have not, as yet, been established in general vacuum spacetimes, some useful insights have nevertheless been gained in studying the potential-modulo-gauge description of the spin- $\frac{3}{2}$ problem in such spacetimes.
\end{abstract}

PACS numbers: 0420, 1115

\section{Introduction}

One of the major research directions of twistor theory in recent years has been to explore the relationship between twistors and the charges of massless spin- $\frac{3}{2}$ fields. First of all, the connection between general relativity and the spin- $\frac{3}{2}$ fields has been known for many years [1]; the consistency conditions for the massless spin- $\frac{3}{2}$ fields in a potential-modulo-gauge description (see discussion below) on a spacetime are that the spacetime must satisfy the vacuum Einstein equations. More recently, it was pointed out by Penrose [2] that in a Minkowski spacetime, the space of the conserved charges of the massless spin- $\frac{3}{2}$ fields is isomorphic to the flat twistor space. This curious result greatly stimulated the search that had already been under way for finding the conserved charges of the spin- $\frac{3}{2}$ fields in general Ricci-flat spacetimes with which to construct the generalized versions of twistor space [3]. If the twistor spaces in question really existed, they would presumably reflect, although in an essentially non-local manner, the properties of the underlying vacuum spacetime; e.g. the vacuum spacetimes would be encoded, in some manner, into the twistor spaces themselves. Unfortunately, this program has not been successful until now.

The present work investigates (by explicit integration) the problem of the charges of the massless spin- $\frac{3}{2}$ fields, first in flat spacetime and then (with the flat-space results as a guide) in asymptotically flat vacuum spacetimes. This procedure allows us to see explicitly how far the flat space results are tenable in the curved spaces and also what the possible obstructions would be. The explicit comparison between the flat and the curved space results may provide a way to envisage the twistor concepts in the curved space case. 
In a Minkowski spacetime, a spin- $\frac{3}{2}$ field, described by a totally symmetric spinor $\psi_{\dot{A} \dot{B} \dot{C}}$, satisfies the zero rest mass field equation

$$
\nabla^{A \dot{A}} \psi_{\dot{A} \dot{B} \dot{C}}=0,
$$

where $\nabla^{A \dot{A}}$ is the spinor-covariant derivative. Throughout the present work, we use twocomponent spinor notation, where spinors with dotted indices transform under the $\left(0, \frac{1}{2}\right)$ representation of the Lorentz group and those with undotted indices under the $\left(\frac{1}{2}, 0\right)$ representation. Here $A=0,1$ and $\dot{A}=\dot{0}, \dot{1}$. In our convention, the spinor field with three symmetric dotted indices represents a spin- $\frac{3}{2}$ particle of positive helicity.

In a general curved spacetime, equation (1.1) is rendered inconsistent by the well known Buchdahl condition:

$$
\Psi_{\dot{A} \dot{B} \dot{C} \dot{D}} \psi^{\dot{A} \dot{B} \dot{C}}=0,
$$

where $\Psi_{\dot{A} \dot{B} \dot{C} \dot{D}}$ is the conformal Weyl spinor. Equation (1.2) is a condition on the spinor field and the spacetime that severely restricts the set of solutions of equation (1.1). In order to circumvent this difficulty, one introduces a potential $\sigma^{C} \dot{A} \dot{B}$, which, in flat space, describes locally the spinor field $\psi_{\dot{A} \dot{B} \dot{C}}$.

In a Minkowski spacetime, two kinds of potential formulations have been given - the Dirac [4] and the Rarita-Schwinger [5] formulations. In the Dirac formulation, the spinor field $\psi_{\dot{A} \dot{B} \dot{C}}$ is defined in terms of a potential $\sigma^{C}{ }_{\dot{A} \dot{B}}$ as

$$
\left.\psi_{\dot{A} \dot{B} \dot{C}} \quad=\nabla_{C(\dot{C}} \sigma^{C} \dot{A} \dot{B}\right),
$$

with

$$
\nabla^{A \dot{A}} \sigma^{C} \dot{A} \dot{B}=0,
$$

where the potential $\sigma^{C} \dot{A} \dot{B}$ is symmetric in the indices $\dot{A}$ and $\dot{B}$. Equation (1.3), together with equation (1.4), implies the validity of equation (1.1).

The gauge transformation for $\sigma^{C} \dot{A} \dot{B}$ which leaves $\psi_{\dot{A} \dot{B} \dot{C}}$ invariant is given by

$$
\sigma_{\dot{A} \dot{B}}^{C} \longrightarrow \sigma^{C}{ }_{\dot{A} \dot{B}}+\nabla^{C}{ }_{\dot{A}} v_{\dot{B}},
$$

where the spin- $\frac{1}{2}$ field $v_{\dot{B}}$ satisfies the Weyl anti-neutrino equation

$$
\nabla^{A \dot{A}} v_{\dot{A}}=0 .
$$

In the Rarita-Schwinger formulation, equation (1.3), which defines the field in terms of the potential, is unchanged. The potential, however, is no longer symmetric in the indices $\dot{A}$ and $\dot{B}$ and now satisfies the Rarita-Schwinger equations

$$
\begin{aligned}
& \nabla_{C\left(\dot{C} \sigma^{C}\right.}{ }_{\dot{A}) \dot{B}} \epsilon^{\dot{A} \dot{B}}=0, \\
& \nabla^{\dot{A}(A} \sigma^{C)} \dot{A} \dot{B} \quad=0 .
\end{aligned}
$$

Equation (1.3), together with the Rarita-Schwinger equations (1.7) and (1.8), implies the validity of equation (1.1).

$\sigma^{C}{ }_{\dot{A} \dot{B}}$ has the same gauge transformation as in equation (1.5), with the exception that $v_{\dot{B}}$ is now completely arbitrary. It may be mentioned in passing that the Rarita-Schwinger formulation yields the Dirac formulation for a special choice of gauge.

It has been shown by Buchdahl [1] and by Chinea [6] among others and later also by people working in supergravity theory $[7,8]$ that the integrability conditions for the potential equations (the Dirac equation, equation (1.4), in the Dirac formulation, and the Rarita-Schwinger equations, equations (1.7) and (1.8), in the Rarita-Schwinger formulation) in an arbitrary curved spacetime are that the traceless part of the Ricci tensor $R_{\mu \nu}$ vanishes. 
Furthermore, if the potential $\sigma^{C} \dot{A} \dot{B}$ has to enjoy the same gauge freedom as that given by equation (1.5), the trace part of $R_{\mu v}$ must vanish, so that the requirement for consistency both of the Dirac and of the Rarita-Schwinger formulation in an underlying curved spacetime is that the spacetime is Ricci-flat, i.e.

$$
R_{\mu \nu}=0 .
$$

It is important, however, to recognize in this connection that any massless spin- $\frac{3}{2}$ field $\psi_{\dot{A} \dot{B} \dot{C}}$ defined from the potentials in either of the two formulations is not gauge invariant, and one cannot help being resigned to a potential-modulo-gauge description.

Frequently, a second potential $\rho_{\dot{A}}^{B C}$ is introduced, which locally defines the first potential $\sigma^{C}{ }_{\dot{A} \dot{B}}$. In the Dirac formulation, the second potential $\rho_{\dot{A}}{ }^{B C}$ is symmetric in the indices $B$ and $C$ and is given by

$$
\sigma_{\dot{A} \dot{B}}^{C}=\nabla_{B(\dot{B}} \rho_{\dot{A})}{ }^{B C},
$$

with

$$
\nabla^{A \dot{A}} \rho_{\dot{A}}^{B C}=0,
$$

subject to the following gauge freedom

$$
\rho_{\dot{A}}^{B C} \longrightarrow \rho_{\dot{A}}^{B C}+\nabla^{B}{ }_{\dot{A}} \chi^{C}+\epsilon^{B C} v_{\dot{A}},
$$

where the spin- $\left(-\frac{1}{2}\right)$ field $\chi^{C}$ satisfies the Weyl neutrino equation

$$
\nabla_{C \dot{C}} \chi^{C}=0 \text {. }
$$

The $\epsilon^{B C} v_{\dot{A}}$ term in the gauge transformation of $\rho_{\dot{A}}^{B C}$ in equation (1.11) generates the correct gauge transformation of the first potential $\sigma^{C} \dot{A} \dot{B}$, as given by equation (1.5). Equations (1.9) and (1.10), along with equations (1.3) and (1.4), imply the validity of equation (1.1).

In the Rarita-Schwinger formulation, the symmetry condition is not imposed on the second potential. The first potential $\sigma^{C}{ }_{\dot{A} \dot{B}}$ is related to the second potential by the following equations:

$$
\begin{aligned}
\sigma_{\dot{A}}^{C \dot{A}} & =-\frac{4}{3} \nabla^{\dot{B}(C} \rho^{B)}{ }_{B \dot{B}}, \\
\sigma_{(\dot{A} \dot{B})}^{C} & =2 \nabla_{B(\dot{A}} \rho_{\dot{B})}{ }^{B C} .
\end{aligned}
$$

With the above defining relations between the two potentials, the first Rarita-Schwinger equation, (1.7), reduces to an identity, while the second Rarita-Schwinger equation, (1.8), yields a second-order equation for $\rho_{\dot{A}}{ }^{B C}$ which can be satisfied by solving the first-order equation

$$
\nabla^{\dot{A}(A} \rho_{\dot{A}}^{B C)}=0 .
$$

The gauge transformation for $\rho_{\dot{A}}^{B C}$ is again given by equation (1.11), but now $\chi^{C}$ is just an arbitrary spinor field.

In a Minkowski spacetime, a consistent identification of twistors with the charges of massless spin- $\frac{3}{2}$ fields has been made [2]. The concept of charge arises here in close analogy with Gauss's law in electrodynamics, where the charge $Q$ of an electromagnetic field is obtained by integrating the field tensor $F_{\mu \nu}$ (a closed 2-form) over a sphere enclosing the source:

$$
Q=\int_{S} F_{\mu \nu} \mathrm{d} x^{\mu \nu}
$$


In order to obtain a generalized Gauss's law to define charges for spin- $\frac{3}{2}$ fields, one begins with a spinor field of the form

$$
\beta^{\dot{A}}=\mu^{\dot{A}}+\mathrm{i} x^{A \dot{A}} \lambda_{A},
$$

(the pair of constant spinors $\left(\lambda_{A}, \mu^{\dot{A}}\right)$ defines a dual twistor $W_{\alpha}$ ) which satisfies the dual twistor equation

$$
\nabla^{A(\dot{A}} \beta^{\dot{B})}=0 .
$$

One then defines the Maxwell spinor

$$
\phi_{\dot{A} \dot{B}}=\psi_{\dot{A} \dot{B} \dot{C}} \beta^{\dot{C}},
$$

which satisfies the self-dual Maxwell equations, and the charge is given by

$$
Q(\beta)=\int_{S} \phi_{\dot{A} \dot{B}} \mathrm{~d} S^{\dot{A} \dot{B}}
$$

where $S$ is any closed 2-surface of the topology of $S^{2}$ enclosing the region of spacetime which contains the sources of the spin- $\frac{3}{2}$ fields. For any $\beta^{\dot{A}}$, one thus obtains a complex number $Q(\beta)$ which depends linearly on the dual twistor $W_{\alpha}$. Given a spin- $\frac{3}{2}$ field $\psi_{\dot{A} \dot{B} \dot{C}}$, one therefore finds a twistor $Q^{\alpha}$ by means of the duality relation $Q(\beta)=Q^{\alpha} W_{\alpha}$. This $Q^{\alpha}$ may be called the charge twistor of the field $\psi_{\dot{A} \dot{B} \dot{C}}$. As $\psi_{\dot{A} \dot{B} \dot{C}}$ ranges over all possible field configurations, one obtains in this way a complete twistor space of charges. This space arises in a way which is completely different from that in which twistors are usually tied in with the geometry of a complexified Minkowski space. It is natural to ask whether one can make a suitable connection between the charge twistor of a spin- $\frac{3}{2}$ field and an $\alpha$-plane that defines a twistor in the more geometric picture. It turns out that this can indeed be done by looking at the asymptotic behaviour of the field and by locating an $\alpha$-curve or a twistor line (i.e. the intersection of an $\alpha$-plane with null infinity $\mathcal{I}$ ) in terms of the field only. This will be described in more detail in a forthcoming paper.

In section 2 we present the asymptotic solution of the massless spin- $\frac{3}{2}$ field equations in flat space and show explicitly how the conserved quantities that appear in the field reappear as singular terms in the two potentials. In section 3, we exhibit a particularly interesting exact solution of the spin- $\frac{3}{2}$ problem, the solution for pure charges or the 'quasi-Coulumb' solution. In section 4, we study the asymptotic solutions of the Rarita-Schwinger equations and also the behaviour of the second potential in Ricci-flat spacetimes. Section 5 considers the possibility of defining conserved quantities as charges in an arbitrary vacuum spacetime. Section 6 summarizes the main results of the present work and also points out some further aspects of the spin- $\frac{3}{2}$ problem that are currently being investigated.

\section{Asymptotic solution in flat spacetime}

In this section we study the asymptotic behaviour of the spin- $\frac{3}{2}$ fields and their associated potentials in Minkowski spacetime. In particular, we find certain conserved quantities (twistors), which are the charged sources of the fields. We then show how these quantities appear in the two potentials.

We first introduce standard null spherical (Bondi) coordinates $(u, r, \zeta, \bar{\zeta})[9,10]$, where $u$ is the retarded time and $r$ the radial distance in the usual system. $\zeta$ and $\bar{\zeta}$ are the standard complex stereographic coordinates on the sphere. The hypersurfaces, $u=$ constant, are the outgoing light cones with vertices on $r=0$. 
At any point in the spacetime, a complex null tetrad or vierbein $\lambda^{\mu}{ }_{a}=\left(l^{\mu}, n^{\mu}, m^{\mu}, \bar{m}^{\mu}\right)$ can be introduced as follows: $l^{\mu}$ is an outward-pointing null vector tangent to the cone $u=$ constant, $n^{\mu}$ is a null vector pointing inward toward $r=0$, and $m^{\mu}$ and $\bar{m}^{\mu}$ are complex vectors tangent to the 2-sphere $S^{2}$ defined by constant $r$ and $u, \bar{m}^{\mu}$ being the complex conjugate of $m^{\mu}$. Here $\mu(\mu=0,1,2,3)$ is the spacetime index, and the index $a$ ( $a=0,1,2,3)$ stands for the name of the vector $\lambda^{\mu}{ }_{a}$. The exact forms of the tetrad can be found in $[9,10]$.

The components of the spin- $\frac{3}{2}$ field in the vierbein basis are given by four complex functions $\phi_{0}, \phi_{1}, \phi_{2}$ and $\phi_{3}$ having spin weights $-\frac{3}{2},-\frac{1}{2}, \frac{1}{2}$ and $\frac{3}{2}$, respectively. These quantities are defined in terms of the spinor field $\psi_{\dot{A} \dot{B} \dot{C}}$ as

$$
\begin{aligned}
& \phi_{0}=\psi_{\dot{0} \dot{0} \dot{0}}=\zeta_{\dot{0}}^{\dot{A}} \zeta_{\dot{0}}^{\dot{B}} \zeta_{\dot{0}}^{\dot{C}} \psi_{\dot{A} \dot{B} \dot{C}}=o^{\dot{A}} o^{\dot{B}} o^{\dot{C}} \psi_{\dot{A} \dot{B} \dot{C}}, \\
& \phi_{1}=\psi_{\dot{0} \dot{0} \dot{1}}=\zeta_{\dot{0}}{ }^{\dot{A}} \zeta_{\dot{0}}{ }^{\dot{B}} \zeta_{\dot{1}}{ }^{\dot{C}} \psi_{\dot{A} \dot{B} \dot{C}}=o^{\dot{A}} o^{\dot{B}} \iota^{\dot{C}} \psi_{\dot{A} \dot{B} \dot{C}},
\end{aligned}
$$

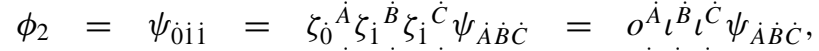

$$
\begin{aligned}
& \phi_{3}=\psi_{i \mathrm{ii}}=\zeta_{\dot{1}}{ }^{\dot{A}} \zeta_{\dot{1}}{ }^{\dot{B}} \zeta_{\dot{1}}{ }^{\dot{C}} \psi_{\dot{A} \dot{B} \dot{C}}=\iota^{\dot{A}} \iota^{\dot{B}} \iota^{\dot{C}} \psi_{\dot{A} \dot{B} \dot{C}},
\end{aligned}
$$

where $\zeta_{\boldsymbol{a}}{ }^{A}=\left(\zeta_{0}{ }^{A}, \zeta_{1}{ }^{A}\right)=\left(o^{A}, \iota^{A}\right)$ (the complex conjugate being $\zeta_{\dot{a}}{ }^{\dot{A}}=\left(\zeta_{0}{ }^{\dot{A}}, \zeta_{\dot{1}}{ }^{\dot{A}}\right)=$ $\left.\left(o^{\dot{A}}, \iota^{\dot{A}}\right)\right)$ is the standard spin-frame or the zweibein associated with the Bondi null tetrad by the following relations [11]:

$$
l^{\mu}=o^{A} o^{\dot{A}}, \quad n^{\mu}=\iota^{A} \iota^{\dot{A}}, \quad m^{\mu}=o^{A} \iota^{\dot{A}}, \quad \bar{m}^{\mu}=\iota^{A} o^{\dot{A}},
$$

with the standard normalization

$$
l^{\mu} n_{\mu}=1, \quad m^{\mu} \bar{m}_{\mu}=-1, \quad o_{A} \iota^{A}=1 .
$$

To be precise, we should have used $\bar{\phi}_{0}, \bar{\phi}_{1}, \bar{\phi}_{2}$ and $\bar{\phi}_{3}$ in lieu of $\phi_{0}, \phi_{1}, \phi_{2}$ and $\phi_{3}$ to define quantities belonging to the complex conjugate space, but to keep our notation uncluttered, we have avoided putting the bars on the $\phi$ 's. The spin- $\frac{3}{2}$ field equation, equation (1.1), can now be expressed in the tetrad basis in terms of the $\phi$ 's by the following set of field equations:

$$
\begin{aligned}
& \left(\frac{\partial}{\partial u}-\frac{1}{2} \frac{\partial}{\partial r}\right) \phi_{0}-\frac{1}{2 r} \phi_{0}+\frac{1}{r} \bar{\partial} \phi_{1}=0, \\
& \frac{1}{r} \partial \phi_{0}+\left(\frac{\partial}{\partial r}+\frac{3}{r}\right) \phi_{1}=0, \\
& \left(\frac{\partial}{\partial u}-\frac{1}{2} \frac{\partial}{\partial r}\right) \phi_{1}-\frac{1}{r} \phi_{1}+\frac{1}{r} \bar{\partial} \phi_{2}=0, \\
& \frac{1}{r} \partial \phi_{1}+\left(\frac{\partial}{\partial r}+\frac{2}{r}\right) \phi_{2}=0, \\
& \left(\frac{\partial}{\partial u}-\frac{1}{2} \frac{\partial}{\partial r}\right) \phi_{2}-\frac{3}{2 r} \phi_{2}+\frac{1}{r} \bar{\partial} \phi_{3}=0, \\
& \frac{1}{r} \partial \phi_{2}+\left(\frac{\partial}{\partial r}+\frac{1}{r}\right) \phi_{3}=0 .
\end{aligned}
$$

The operators, edth and edthbar $(\check{\partial}$ and $\bar{\delta})$ are angular differential operators which act on spin-weighted functions. Their definitions can be found in $[10,11]$.

If $\phi_{0}$ is given on one light cone at $u=u_{0}$ as an arbitrary function of $r, \zeta$, and $\bar{\zeta}$, equations (2.3), (2.5), and (2.7) can at once be integrated by treating $r$ as an independent variable and $\zeta$ and $\bar{\zeta}$ as parameters. 
To establish good 'peeling behaviour' in the solutions of the above equations, we assume

$$
\phi_{0}=\frac{\stackrel{(0)}{\phi_{0}}}{r^{4}}+\frac{\stackrel{(1)}{\phi}}{r^{5}}+O\left(r^{-6}\right) .
$$

Then, from equations (2.3), (2.5) and (2.7) we obtain

$$
\begin{aligned}
& \phi_{1}=\frac{\stackrel{(0)}{\phi_{1}}}{r^{3}}+\frac{1}{r^{4}} \partial \stackrel{(0)}{\phi_{0}}+\mathrm{O}\left(r^{-5}\right), \\
& \phi_{2}=\frac{\stackrel{(0)}{\phi_{2}}}{r^{2}}+\frac{1}{r^{3}} \partial \stackrel{(0)}{\phi_{1}}+\mathrm{O}\left(r^{-4}\right), \\
& \phi_{3}=\frac{\stackrel{(0)}{\phi_{3}}}{r}+\frac{1}{r^{2}} \partial \stackrel{(0)}{\phi_{2}}+\mathrm{O}\left(r^{-3}\right),
\end{aligned}
$$

Since there is no equation which governs the $u$ dependence of $\stackrel{(0)}{\phi}$, it can be given as a free function of $u, \zeta$ and $\bar{\zeta} . \stackrel{(0)}{\phi_{3}}$ is commonly known as the 'news function' in the literature. With the above $r$-dependence of the leading terms in the $\phi$-expansions, equations (2.2), (2.4) and (2.6) now yield, at the lowest order,

$$
\begin{aligned}
& \frac{\partial \stackrel{(0)}{\phi}}{\partial u}=-\bar{\varnothing} \stackrel{(0)}{\phi}, \\
& \frac{\partial \stackrel{(0)}{\phi}}{\partial u}=-\bar{\partial} \stackrel{(0)}{\phi}_{2}, \\
& \frac{\partial \stackrel{(0)}{\phi}}{\partial u}=-\bar{\varnothing} \stackrel{(0)}{\phi}{ }_{1} .
\end{aligned}
$$

The solutions of these equations are given by

$$
\begin{aligned}
& \stackrel{(0)}{\phi_{2}}=C_{2}+D_{2}-\int_{-\infty}^{u} \mathrm{~d} u^{\prime} \overline{\bar{\delta}} \stackrel{(0)}{\phi_{3}}, \\
& \stackrel{(0)}{\phi}{ }_{1}=C_{1}+D_{1}-u \bar{\varnothing} C_{2}-u \bar{\partial} D_{2}+\int_{-\infty}^{u} \int_{-\infty}^{u^{\prime}} \mathrm{d} u^{\prime} \mathrm{d} u^{\prime \prime} \bar{\varnothing}^{2} \stackrel{(0)}{\phi_{3}} \text {, } \\
& \stackrel{(0)}{\phi}_{0}=D_{0}-u \bar{\partial} D_{1}+\frac{u^{2}}{2} \bar{\delta}^{2} D_{2}-\int_{-\infty}^{u} \int_{-\infty}^{u^{\prime}} \int_{-\infty}^{u^{\prime \prime}} \mathrm{d} u^{\prime} \mathrm{d} u^{\prime \prime} \mathrm{d} u^{\prime \prime \prime} \bar{\delta}^{3} \stackrel{(0)}{\phi_{3}} \text {. }
\end{aligned}
$$

Here

$$
C_{2}=\sum_{m} \beta_{m} Y_{\frac{1}{2}} Y_{\frac{1}{2} m}, \quad m=-\frac{1}{2}, \frac{1}{2}
$$

and

$$
C_{1}=\sum_{m} \delta_{m-\frac{1}{2}} Y_{\frac{1}{2} m}, \quad m=-\frac{1}{2}, \frac{1}{2},
$$

where $\beta_{m}$ and $\delta_{m}$ are four constants, the 'charges' of the spin- $\frac{3}{2}$ fields.

In equations (2.15) and (2.16), there is the possibility that the initial values of ${ }^{(0)} \quad{ }_{0}^{(0)}$ and $\stackrel{(0)}{\phi_{2}}$ may contain terms having $l$ values higher than $l=\frac{1}{2}$. The $u$-independent (i.e. constant) quantities $C_{2}$ and $C_{1}$ are the $l=\frac{1}{2}$ components of the initial values, while the 
other (constant) components having $l$ values higher than $l=\frac{1}{2}$ are lumped together in the sum of terms $D_{2}$ and $D_{1}$. This decomposition facilitates exhibiting the 'twistor charges' of the spin- $\frac{3}{2}$ fields in terms of the four complex conserved quantities $\beta_{m}$ and $\delta_{m}$. However, by suitably choosing the values of the field $\stackrel{(0)}{\phi}$ on the initial hypersurface $u=-\infty$, we can set $D_{0}, D_{1}$ and $D_{2}$ equal to zero. We shall henceforth be concerned only with the four complex conserved quantities $C_{1}$ and $C_{2}$. For each value of $s,{ }_{s} Y_{l m}$ are the spin-weighted spherical harmonics forming a complete orthonormal set for all spin-weighted functions. Their properties can be found in $[10,11]$.

Since the asymptotic $\phi$ 's are completely known, the potentials via equation (1.3) can now be obtained in terms of the fields. This allows us to explicitly see the conserved charges in certain components of the potentials. Throughout this work we deal exclusively with the Rarita-Schwinger description of the potentials, though by a gauge transformation the Dirac solution can be obtained.

A remark concerning the status of the field-potential relations (1.3) and the RaritaSchwinger equations (1.7) and (1.8) is appropriate here. It is important to recognize that the field-potential relations alone cannot yield all the components of the potential uniquely. Even after the relations have been integrated, some components of the potential, which are not pure gauge, will remain undetermined in the solution. In order to obtain these quantities, one must appeal to the Rarita-Schwinger equations, which are stronger conditions on the solution set of the potential than the field-potential relations. On the other hand, the potential, which does not naturally 'peel', enjoys some of the peeling properties of the field through the field-potential relations only. This becomes a problem when we study the Rarita-Schwinger equations in curved vacuum (Ricci-flat) spacetime in which there exists no gauge invariant spin- $\frac{3}{2}$ field $\psi_{\dot{A} \dot{B} \dot{C}}$ to start with.

Before integrating the potential equations, we must settle the issue of the gauge fixing of the potential. In the first place, we note that the field-potential relations (1.3) constitute a set of four equations involving the four components $\phi_{0}, \phi_{1}, \phi_{2}$ and $\phi_{3}$ of $\psi_{\dot{A} \dot{B} \dot{C}}$ and the eight tetrad components, $\sigma^{0}{ }_{00}, \sigma^{0}{ }_{0} 1, \ldots, \sigma^{1}{ }_{i i}$ of the potential $\sigma^{C}{ }_{\dot{A} \dot{B}}$ defined as follows:

$$
\begin{aligned}
& \sigma^{0} \dot{0}=\zeta_{A}{ }^{0} \zeta_{\dot{0}} \dot{B}_{\dot{0}}{ }_{\dot{0}}{ }^{\dot{C}} \sigma^{A} \dot{B} \dot{C}=-\iota_{A} o^{\dot{B}} O^{\dot{C}} \sigma^{A}{ }_{\dot{B} \dot{C}}, \\
& \sigma^{0}{ }_{0 \mathrm{i}}=\zeta_{A}{ }^{0} \zeta_{0}{ }^{\dot{B}} \zeta_{\dot{1}}{ }^{\dot{C}} \sigma^{A}{ }_{\dot{B} \dot{C}}=-\iota_{A} o^{\dot{B}} O^{\dot{C}} \sigma^{A}{ }_{\dot{B} \dot{C}},
\end{aligned}
$$

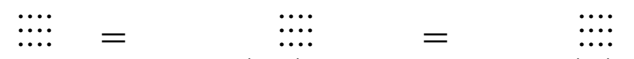

$$
\begin{aligned}
& \sigma^{1}{ }_{\mathrm{ii}}=\zeta_{A}{ }^{1} \zeta_{\mathrm{i}}{ }^{\dot{B}} \zeta_{\dot{1}} \dot{C}_{\sigma^{A}}{ }_{\dot{B} \dot{C}}=o_{A} \iota^{\dot{B} \iota} \iota^{\dot{C}} \sigma^{A} \dot{B} \dot{C} .
\end{aligned}
$$

We can set two of the eight components of the potential equal to zero by imposing the null gauge condition:

$$
l^{\mu} \sigma_{\mu \dot{A}}=0
$$

or

$$
\sigma^{1} \dot{0} \dot{0}=0=\sigma^{1} \dot{\dot{1}}
$$

so that there are only six independent components of the potential. However, even after imposing the null gauge, there still remains some residual gauge freedom in the choice of the potential. This freedom will be used to eliminate some of the constants of integration in the solution.

Before we start integrating the potential equations asymptotically (for known fields $\left.\psi_{\dot{A} \dot{B} \dot{C}}\right)$, it is worthwhile to present some exact relations between the various components of $\sigma^{C}{ }_{\dot{A} \dot{B}}$ which can be deduced directly from the Rarita-Schwinger equations. These are

$$
\sigma^{1}{ }_{\mathrm{i} 0}=\frac{\stackrel{(1)}{\sigma}^{1} \mathrm{i} \dot{0}}{r}=0,
$$




$$
\begin{aligned}
& \sigma^{1}{ }_{\mathrm{ii}}=\frac{\stackrel{(1)}{\sigma}{ }_{\mathrm{ii}}}{r}, \\
& \left(\frac{\partial}{\partial r}+\frac{2}{r}\right) \sigma^{0}{ }_{0} \mathrm{i}=-\frac{1}{r} \partial \sigma^{0} \dot{0}, \\
& \frac{\partial}{\partial r} \sigma^{0}{ }_{i \dot{ }}=\left(\frac{\partial}{\partial r}+\frac{1}{r}\right) \sigma^{0}{ }_{0 \mathrm{i}}, \\
& \left(\frac{\partial}{\partial r}+\frac{1}{r}\right) \sigma^{0}{ }_{i i}=-\frac{1}{r} \partial \sigma^{0}{ }_{i 0}, \\
& \frac{1}{r} \bar{\partial}{ }_{\sigma}^{(1)}{ }_{i i}+\partial \sigma_{0 i}^{0}-\partial \sigma_{i 0}^{0}-\sigma^{0}{ }_{i i}=0 .
\end{aligned}
$$

In equation (2.23), $\sigma^{1}$ io has been set equal to zero by gauge choice (see discussion below). The above relations prove to be very useful in simplifying the asymptotic solutions of $\sigma^{C} \dot{A} \dot{B}$ in terms of the fields $\psi_{\dot{A} \dot{B} \dot{C}}$ given below.

The radial expansion of the tetrad components of the potential $\sigma^{C} \dot{A} \dot{B}$, which is found from the radial behaviour of the $\phi$ 's, is given by

$$
\begin{aligned}
& \sigma_{\dot{0} \dot{0}}^{0}=\frac{\stackrel{(1)}{\sigma} 0 \dot{0} \dot{0}}{r}+\frac{\stackrel{(2)}{\sigma} 0 \dot{0} \dot{0}}{r^{2}}+\mathrm{O}\left(r^{-3}\right), \\
& \sigma_{0 \mathrm{i}}^{0}=\frac{\stackrel{(1)}{\sigma} 0_{0} \mathrm{i}}{r}+\frac{\stackrel{(2)}{\sigma} 0_{0} \mathrm{i}}{r^{2}}+\mathrm{O}\left(r^{-3}\right),
\end{aligned}
$$

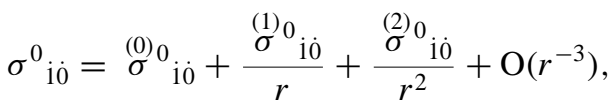

$$
\begin{aligned}
& \sigma^{0}{ }_{\mathrm{ii}}=\stackrel{(0)}{\sigma}_{0} \mathrm{ii}+\frac{\stackrel{(1)}{\sigma}^{0} \mathrm{ii}}{r}+\frac{\stackrel{(2)}{\sigma}_{0} \mathrm{ii}}{r^{2}}+\mathrm{O}\left(r^{-3}\right), \\
& \sigma^{1}{ }_{\mathrm{i} 0}=\frac{\stackrel{(1)}{\sigma} 1 \mathrm{i} \dot{0}}{r}+\frac{\stackrel{(2)}{\sigma} 1 \mathrm{i} \dot{\mathrm{i}}}{r^{2}}+\mathrm{O}\left(r^{-3}\right) \text {, } \\
& \sigma^{1}{ }_{\mathrm{ii}}=\frac{\stackrel{(1)}{\sigma}{ }_{\mathrm{ii}}}{r}+\frac{\stackrel{(2)}{\sigma}{ }_{\mathrm{ii}}}{r}+\mathrm{O}\left(r^{-3}\right) \text {. }
\end{aligned}
$$

By comparing the coefficients of the different powers of $\frac{1}{r}$ in the tetrad version of equation (1.3), we obtain a series of relations between the tetrad components of $\stackrel{(n)}{\sigma} A_{\dot{B} \dot{C}}$ and the $\phi$ 's. In particular, we find

$$
\frac{\partial \stackrel{(1)}{\sigma}{ }_{1} \dot{10}}{\partial u}=0
$$

or

$$
\stackrel{(1)}{\sigma}^{1}{ }_{\mathrm{i} 0}=G_{0}(\zeta, \bar{\zeta})
$$

where $G_{0}(\zeta, \bar{\zeta})$ is an arbitrary function of $\zeta$ and $\bar{\zeta}$, and

or

$$
\frac{\partial \stackrel{(1)}{\sigma}{ }_{1} \mathrm{ii}}{\partial u}=\stackrel{(0)}{\phi},
$$

$$
\stackrel{(1)}{\sigma}_{1}{ }_{\mathrm{ii}}=I_{0}(\zeta, \bar{\zeta})+\int_{-\infty}^{u} \mathrm{~d} u^{\prime} \stackrel{(0)}{\phi}_{3}
$$


We soon return to these two relations.

The gauge transformations of $\sigma^{1}{ }_{\dot{0} \dot{0}}$ and $\sigma^{1}{ }_{\dot{0} \mathrm{i}}$ are given by

$$
\sigma^{1}{ }_{\dot{0} \dot{0}} \longrightarrow \sigma^{1} \dot{0} \dot{0}-\frac{\partial v_{\dot{0}}}{\partial r}
$$

and

$$
\sigma^{1}{ }_{\mathrm{oi}} \longrightarrow \sigma^{1}{ }_{\mathrm{oi}}-\frac{\partial v_{\mathrm{i}}}{\partial r}
$$

The preservation of the null gauge condition $\left(\sigma^{1}{ }_{0 \dot{0}}=0=\sigma^{1} \dot{ } \dot{\mathrm{i}}\right)$ therefore implies that

$$
\frac{\partial v_{0}}{\partial r}=0=\frac{\partial v_{i}}{\partial r}
$$

so that in the remaining gauge freedom $v_{0}$ and $v_{1}$ are functions of $u, \zeta$ and $\bar{\zeta}$ only. Next, the $r$-independent terms in the $\sigma^{0}{ }_{i 0}$ and $\sigma^{0}{ }_{i i}$ series can be made to vanish by making use of the residual gauge freedom leaving as gauge freedom the two functions $v_{0}$ and $v_{i}$ depending on $\zeta$ and $\bar{\zeta}$ only. Furthermore, the gauge transformations of the components $\stackrel{(1)}{\sigma}{ }^{1}$ io and $\stackrel{(1)}{\sigma}{ }_{\text {ii }}$ are given by

$$
\stackrel{(1)}{\sigma}{ }_{\mathrm{i} 0} \longrightarrow \stackrel{(1)}{\sigma}^{1}{ }_{\mathrm{i} 0}+v_{\mathrm{i}}+\partial v_{0}
$$

and

$$
\stackrel{(1)}{\sigma}{ }_{\mathrm{ii}} \longrightarrow \stackrel{(1)}{\sigma}{ }_{\mathrm{ii}}+\partial v_{\mathrm{i}}
$$

This residual gauge freedom allows us to set both $G_{0}$ and $I_{0}$ equal to zero. In the remainder we thus have $G_{0}=I_{0}=0$ as well as $\stackrel{(0)}{\sigma}^{\circ}{ }_{\mathrm{i} 0}=\stackrel{(0)}{\sigma}_{0}{ }_{\mathrm{ii}}=0$.

With the above simplifications, the relevant information for the leading terms of the tetrad components of the potential $\sigma^{C} \dot{A} \dot{B}$, obtained from equation (1.3) in conjunction with the Rarita-Schwinger equations (1.7) and (1.8), is

$$
\begin{aligned}
& \frac{\partial \stackrel{(1)}{\sigma} 0 \dot{0} \dot{0}}{\partial u}=0=\frac{\partial \stackrel{(1)}{\sigma} 0 \dot{0} \mathrm{i}}{\partial u}, \\
& \partial^{2} \stackrel{(1)}{\sigma}_{0}{ }_{00}=-\partial \stackrel{(1)}{\sigma}{ }_{0} \mathrm{i}=C_{2}, \\
& \sigma^{1}{ }_{\mathrm{i} 0}=\frac{\stackrel{(1)}{\sigma}^{1} \mathrm{i} \dot{0}}{r}=0 \text {, } \\
& \sigma^{1}{ }_{\mathrm{ii}}=\frac{\stackrel{(1)}{\sigma}{ }_{\mathrm{ii}}}{r}, \\
& \stackrel{(1)}{\sigma}^{1} \mathrm{ii}=\int_{-\infty}^{u} \mathrm{~d} u^{\prime} \stackrel{(0)}{\phi_{3}} \text {, } \\
& \stackrel{(1)}{\sigma}{ }_{\mathrm{i} 0}=0=\stackrel{(2)}{\sigma}_{0} 0 \text { } 0 \text {, } \\
& \stackrel{(1)}{\sigma}^{0} \mathrm{ii}=-\stackrel{(0)}{\phi}_{2}=-C_{2}+\int_{-\infty}^{u} \mathrm{~d} u^{\prime} \overline{\bar{\delta}} \stackrel{(0)}{\phi}_{3} .
\end{aligned}
$$

The other conserved quantity $C_{1}$ appears in $\sigma^{0}{ }_{01}$ as

$$
-\stackrel{(2)}{\sigma}_{0}{ }_{0} \mathrm{i}=\stackrel{(0)}{\phi}_{1}=C_{1}-u \bar{\delta} C_{2}+\int_{-\infty}^{u} \int_{-\infty}^{u^{\prime}} \mathrm{d} u^{\prime} \mathrm{d} u^{\prime \prime} \overline{\bar{\partial}}^{2} \stackrel{(0)}{\phi}_{3} .
$$

There is an important issue that we should discuss now. Let us focus our attention on equation (2.37), which is

$$
\partial^{2} \stackrel{(1)}{\sigma}{ }_{0 \dot{0}}=C_{2} \text {. }
$$


Since $\partial$ is a spin-raising operator (in steps of unity), and since $\stackrel{(1)}{\sigma}_{0}{ }_{00}$ has spin weight $-\frac{3}{2}$, we see from the above equation that the $\delta$ operator cannot supply a non-zero $l=\frac{1}{2}$ part so as to produce the corresponding $l=\frac{1}{2}$ part of $C_{2}$. The equation, therefore, is not soluble for any regular function $\stackrel{(1)}{\sigma}_{00}$ of $\zeta$ and $\bar{\zeta}$ defined globally on the sphere. $\stackrel{(1)}{\sigma}_{0} a_{00}$ possesses an angular singularity similar to the well known string singularity of the Dirac magnetic monopole in Maxwell's electrodynamics (with magnetic monopoles). The sphere must be covered by (at least two) overlapping patches and ${ }_{\sigma}^{(1)} 0_{j 0}$ defined separately on each patch, where it is regular. In the region of overlap of the patches, ${ }_{\sigma}^{(1)} 0_{00}$ are related by gauge transformations. Thus the singularity of $\underset{\sigma}{(1)} 0_{0}$ appearing in one patch of the sphere can be shifted to another by choosing a solution of the homogeneous equation

$$
\partial^{2} \stackrel{(1)}{\sigma_{(H)}}{ }_{0}^{0} \dot{0}=0
$$

which is tantamount to choosing an appropriate singular gauge function in the gauge transformation of $\stackrel{(1)}{\sigma} 0_{j 0}$ that cancels the singularity of $\stackrel{(1)}{\sigma} 0_{00}$ in the patch containing the singularity and moves it to another patch. Explicitly, $\stackrel{(1)}{\sigma}_{0}{ }_{00}$ is given in some patch $(a)$ by

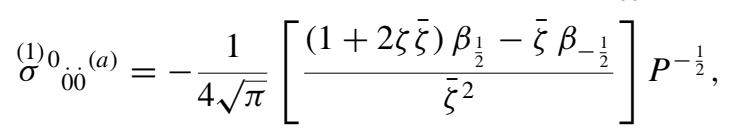

where $P=\frac{1}{2}(1+\zeta \bar{\zeta}) . \beta_{\frac{1}{2}}$ and $\beta_{-\frac{1}{2}}$ are the constants appearing in $C_{2}$ in equation (2.18). ${ }_{\sigma}^{(1)}{ }_{00}$ is clearly singular at $\zeta=0$. Now the general solution of the homogeneous equation (2.44) is given by

$$
\stackrel{(1)}{\sigma_{(H)}} \underset{0}{0} \dot{0}=f(\bar{\zeta}) P^{\frac{3}{2}}
$$

where $f(\bar{\zeta})$ is an arbitrary anti-holomorphic function of integration. Choosing

$$
f(\bar{\zeta})=\frac{1}{\sqrt{\pi}}\left(\frac{\beta_{\frac{1}{2}}}{\bar{\zeta}^{2}}-\frac{\beta_{-\frac{1}{2}}}{\bar{\zeta}}\right)
$$

the singularity at $\zeta=0$ can be removed, so that now in another patch $(b)$

$$
\stackrel{(1)}{\sigma}_{\dot{0} \dot{0}}^{(b)}=\frac{1}{4 \sqrt{\pi}}\left[\zeta^{2} \beta_{\frac{1}{2}}-\zeta(2+\zeta \bar{\zeta}) \beta_{-\frac{1}{2}}\right] P^{-\frac{1}{2}} .
$$

${ }_{\sigma}^{(1)}{ }_{0} \dot{0}^{(b)}$ is singular at $\zeta=\infty$. Patches $(a)$ and $(b)$ of $S^{2}$ are chosen to be $S^{2}-\{0\}$ and $S^{2}-\{\infty\}$ respectively, so that $\stackrel{(1)}{\sigma}_{0 ; 0}{ }^{(a)}$ and $\stackrel{(1)}{\sigma}_{0} 0_{00}{ }^{(b)}$ are both regular in their own domain. The singular gauge function $v_{\dot{A}}$ that shifts the singularity from patch $(a)$ to patch $(b)$ is given by

and

$$
v_{0}=\frac{1}{4 \sqrt{\pi}}\left[\left(\frac{1}{\bar{\zeta}}-\zeta \log \bar{\zeta}\right) \beta_{\frac{1}{2}}+(1+\zeta \bar{\zeta}+\log \bar{\zeta}) \beta_{-\frac{1}{2}}\right] P^{-\frac{1}{2}}
$$

$$
v_{i}=\frac{1}{8 \sqrt{\pi}}\left[(1+\log \bar{\zeta}) \beta_{\frac{1}{2}}+\bar{\zeta} \log \bar{\zeta} \beta_{-\frac{1}{2}}\right] P^{-\frac{1}{2}} .
$$

It is not difficult to verify that with the above singular gauge functions $v_{0}$ and $v_{i}$, no singularities are introduced in any of the regular potential components $\sigma^{0}{ }_{01}, \ldots, \sigma^{1}{ }_{i 1}$, i.e. their analytic structure on $S^{2}$ remains unaffected by this choice of $v_{0}$ and $v_{i}$. A covariant description of the singularities of the potentials and the associated singular gauge transformations is given in section 3 in connection with a particularly simple and exact solution of the spin- $\frac{3}{2}$ equations - the 'quasi-Coulomb' solution. 
Our next task is to use the tetrad versions of equations (1.13) and (1.14) to find the solution for the eight components, $\rho_{0}^{00}, \rho_{0}{ }^{01}, \ldots, \rho_{1}{ }^{11}$ of the second potential $\rho_{\dot{A}}{ }^{B C}$. These quantities are defined as follows:

$$
\begin{aligned}
& \rho_{0}^{00}=\zeta_{\dot{0}}^{\dot{A}} \zeta_{B}{ }^{0} \zeta_{C}{ }^{0} \rho_{\dot{A}}{ }^{B C}=o^{\dot{A}} \iota_{B} \iota_{C} \rho_{\dot{A}}{ }^{B C},
\end{aligned}
$$

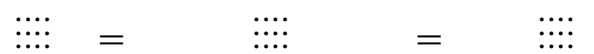

$$
\begin{aligned}
& \rho_{1}{ }^{11}=\zeta_{\dot{1}}^{\dot{A}} \zeta_{B}{ }^{1} \zeta_{C}{ }^{1} \rho_{\dot{A}}{ }^{B C}=\iota^{\dot{A}} o_{B} o_{C} \rho_{\dot{A}}{ }^{B C} .
\end{aligned}
$$

Our procedure here is straightforward and mimics that of the first potential.

First, from the null gauge condition, two of the eight tetrad components of $\rho_{\dot{A}}{ }^{B C}$ are set equal to zero. Explicitly,

$$
l^{\mu} \rho_{\mu}{ }^{A}=0 \Longrightarrow \rho_{0}{ }^{10}=0=\rho_{0}{ }^{11} .
$$

There are thus six independent components of the second potential also. They have the following radial expansion obtained from equations (1.13) and (1.14):

$$
\begin{aligned}
& \rho_{0}^{00}=\stackrel{(0)}{\rho}_{0}^{00}+\frac{\stackrel{(1)}{\rho}_{0}{ }_{0}^{00}}{r}+\frac{\stackrel{(2)}{\rho}_{0}{ }_{0}}{r^{2}}+\mathrm{O}\left(r^{-3}\right), \\
& \rho_{0}^{01}=\frac{\stackrel{(1)}{\rho_{0}}{ }_{0}^{01}}{r}, \\
& \rho_{\mathrm{i}}^{00}=\stackrel{(0)}{\rho}{ }_{\mathrm{i}}^{00}+\frac{\stackrel{(1)}{\rho}{ }_{\mathrm{i}}^{00}}{r}+\frac{\stackrel{(2)}{\rho}_{\mathrm{i}}{ }^{00}}{r^{2}}+\mathrm{O}\left(r^{-3}\right), \\
& \rho_{\mathrm{i}}^{01}=\stackrel{(0)}{\rho}{ }_{\mathrm{i}}^{01}+\frac{\stackrel{(1)}{\rho}{ }_{\mathrm{i}}^{01}}{r}+\frac{\stackrel{(2)}{\rho}_{\mathrm{i}}{ }^{01}}{r^{2}}+\mathrm{O}\left(r^{-3}\right), \\
& \rho_{\mathrm{i}}{ }^{10}=\frac{\stackrel{(1)}{\rho}_{\mathrm{i}}{ }^{10}}{r}+\frac{\stackrel{(2)}{\rho}_{\mathrm{i}} 10}{r^{2}}+\mathrm{O}\left(r^{-3}\right), \\
& \rho_{\mathrm{i}}{ }^{11}=\frac{\stackrel{(1)}{\rho}_{\mathrm{i}}{ }^{11}}{r}+\frac{\stackrel{(2)}{\rho}_{\mathrm{i}}{ }^{11}}{r^{2}}+\mathrm{O}\left(r^{-3}\right) .
\end{aligned}
$$

By imposing the null gauge, the gauge fields $\chi^{0}$ and $\chi^{1}$ are reduced to functions of $u, \zeta$, and $\bar{\zeta}$. The $r$-independent terms in the $\rho_{\mathrm{i}}{ }^{00}$ and $\rho_{\mathrm{i}}{ }^{01}$ series can be made to vanish by the residual gauge choice, thereby restricting the gauge freedom to two functions $\chi^{0}$ and $\chi^{1}$ depending on $\zeta$ and $\bar{\zeta}$ only.

From the tetrad version of equations (1.13) and (1.14), we first note that

$$
\frac{\partial \stackrel{(1)}{\rho}{ }_{\mathrm{i}}{ }^{11}}{\partial u}=\frac{1}{2} \sigma_{\mathrm{ii}}^{1}=\frac{1}{2} \int_{-\infty}^{u} \mathrm{~d} u^{\prime} \stackrel{(\stackrel{0}{\phi})}{\phi_{3}}
$$

or,

$$
\stackrel{(1)}{\rho}{ }_{i}^{11}=J_{0}(\zeta, \bar{\zeta})+\frac{1}{2} \int_{-\infty}^{u} \int_{-\infty}^{u} \mathrm{~d} u^{\prime} \mathrm{d} u^{\prime \prime} \stackrel{(0)}{\phi_{3}},
$$

and

$$
2 \frac{\partial \stackrel{(1)}{\rho}{ }_{\mathrm{i}}{ }^{10}}{\partial u}=\stackrel{(1)}{\sigma}^{0}{ }_{\mathrm{ii}}=-C_{2}+\int_{-\infty}^{u} \mathrm{~d} u^{\prime} \overline{\bar{\partial}} \stackrel{(0)}{\phi_{3}},
$$

or,

$$
\stackrel{(1)}{\rho}{ }_{\mathrm{i}}^{10}=H_{0}(\zeta, \bar{\zeta})-\frac{u}{2} C_{2}+\frac{1}{2} \int_{-\infty}^{u} \int_{-\infty}^{u} \mathrm{~d} u^{\prime} \mathrm{d} u^{\prime \prime} \overline{\bar{\partial}} \stackrel{(0)}{\phi}
$$


As before, we make use of the residual gauge freedom to set both $J_{0}$ and $H_{0}$ equal to zero. The final information for the leading terms in the tetrad components of the second potential $\rho_{\dot{A}}^{B C}$ is now contained in the following results:

$$
\begin{aligned}
& \stackrel{(0)}{\rho}{ }_{0}^{00}=\frac{1}{2} \stackrel{(1)}{\sigma}{ }_{00} \\
& \partial^{2} \stackrel{(1)}{\rho_{0}}{ }_{0}^{00}=\frac{1}{2} \partial C_{1}+\frac{u}{4} C_{2}, \\
& \stackrel{(1)}{\rho}{ }_{0}^{01}=-\frac{u}{2} \partial \stackrel{(1)}{\sigma}{ }_{0} \dot{0}, \\
& \stackrel{(1)}{\rho}{ }_{i} 01=\stackrel{(2)}{\rho}{ }_{i}{ }^{01}=\stackrel{(2)}{\rho}_{i}{ }^{10}=0, \\
& \stackrel{(1)}{\rho} \underset{\mathrm{i}}{00}=-\frac{1}{4} \stackrel{(2)}{\sigma}_{0 \mathrm{i}}=\frac{1}{4} C_{1}-\frac{u}{4} \bar{\partial} C_{2}+\frac{1}{4} \int_{-\infty}^{u} \int_{-\infty}^{u^{\prime}} \mathrm{d} u^{\prime} \mathrm{d} u^{\prime \prime} \bar{\partial}^{2} \stackrel{(0)}{\phi}_{3}, \\
& \stackrel{(1)}{\rho}{ }_{\mathrm{i}}^{10}=-\frac{u}{2} C_{2}+\frac{1}{2} \int_{-\infty}^{u} \int_{-\infty}^{u^{\prime}} \mathrm{d} u^{\prime} \mathrm{d} u^{\prime \prime} \overline{\bar{\partial}} \stackrel{(0)}{\phi}_{3} \text {, } \\
& \stackrel{(1)}{\rho}_{\mathrm{i}}^{11}=\frac{1}{2} \int_{-\infty}^{u} \int_{-\infty}^{u^{\prime}} \mathrm{d} u^{\prime} \mathrm{d} u^{\prime \prime} \stackrel{(0)}{\phi_{3}} \text {. }
\end{aligned}
$$

Equation (2.57) reveals an angular singularity in the $\stackrel{(0)}{\rho}_{0} 00$ (spin weight $=-\frac{3}{2}$ ) component of the second potential. As before, a regular $\stackrel{(0)}{\rho}_{0} 00$ can be defined in a patch of the sphere by removing the singularity from that patch by choosing an appropriate singular gauge function in the gauge transformation of $\stackrel{(0)}{\rho}_{0} 00$. Another angular singularity appears in the potential component $\stackrel{(1)}{\rho}_{0}{ }_{0} 00$ (spin weight $=-\frac{3}{2}$ ), as is evident from equation (2.58). In this case, both the conserved quantities $C_{1}$ and $C_{2}$ contribute to the singularity, so that even if $C_{2}$ were zero from the very outset, $C_{1}$ alone would give rise to the singularity in $\stackrel{(1)}{\rho}{ }_{0}^{00}$. The solution to equation $(2.58)$ on patch $(a)$ is given by

$$
\stackrel{(1)}{\rho_{0}}{ }_{0}^{00}=-\frac{1}{4 \sqrt{\pi}}\left[\frac{(1+2 \zeta \bar{\zeta})\left(\frac{1}{2} \delta_{\frac{1}{2}}+\frac{u}{4} \beta_{\frac{1}{2}}\right)-\bar{\zeta}\left(\frac{1}{2} \delta_{-\frac{1}{2}}+\frac{u}{4} \beta_{-\frac{1}{2}}\right)}{\bar{\zeta}^{2}}\right] P^{-\frac{1}{2}},
$$

which is seen to be singular at $\zeta=0$. This is again an unphysical angular singularity and therefore can be moved to a different patch (not containing the point $\zeta=0$ ) of the sphere by a gauge transformation (see section 3 for details).

In the next section we present an exact solution of the spin- $\frac{3}{2}$ equations; the 'quasiCoulomb' solution. This solution is simple and interesting and possesses all the essential features needed for the purpose of understanding the connection between the conserved quantities (charges) appearing in the solution of the spin- $\frac{3}{2}$ fields and the theory of twistors.

\section{The 'quasi-Coulomb' solution}

In this section we display an exact solution of the spin- $\frac{3}{2}$ equations. This solution is analogous to the Coulomb or monopole solution of Maxwell's electrodynamics; hence the name 'quasi-Coulomb' solution. This is an exact solution obtained by setting the radiation parts of the field equal to zero. It is given by

$$
\phi_{0}=0 \text {, }
$$




$$
\begin{array}{rlrl}
\phi_{1} & =\frac{\stackrel{(0)}{\phi_{1}}}{r^{3}}, & \text { where } \stackrel{(0)}{\phi_{1}}=C_{1}-u \bar{\partial} C_{2}, \\
\phi_{2} & =\frac{\stackrel{(0)}{\phi_{2}}}{r^{2}}+\frac{\partial \stackrel{(0)}{\phi_{1}}}{r^{3}}, & \text { where } \stackrel{(0)}{\phi_{2}}=C_{2}, \\
\phi_{3} & =0 . & &
\end{array}
$$

As in section 2, $C_{2}=\sum_{m} \beta_{m \frac{1}{2}} Y_{\frac{1}{2} m}, m=-\frac{1}{2}, \frac{1}{2}$ and $C_{1}=\sum_{m} \delta_{m}-\frac{1}{2} Y_{\frac{1}{2} m}, m=-\frac{1}{2}, \frac{1}{2}$, where $\beta_{m}$ and $\delta_{m}$ are again the four complex constants, the 'charges' of the spin- $\frac{3}{2}$ field $\psi_{\dot{A} \dot{B} \dot{C}}$. In order to exhibit the conserved quantities in the components of the potential $\sigma^{C} \dot{A} \dot{B}$, we solve the field-potential relations and the Rarita-Schwinger equations exactly. The final solution in null gauge $\left(\sigma^{1} \dot{0} \dot{0}=0=\sigma^{1} \dot{0} \dot{1}\right)$ is given by

$$
\sigma_{\dot{0} \dot{0}}^{0}=\frac{\stackrel{(1)}{\sigma}_{\dot{0} \dot{0}}(\zeta, \bar{\zeta})}{r}
$$

where

$$
\begin{aligned}
& \check{\partial}^{2} \stackrel{(1)}{\sigma}_{0}^{0} \dot{0}=C_{2}, \\
& \sigma_{\dot{0} \mathrm{i}}^{0}=-\frac{\partial \stackrel{(1)}{\sigma}{ }_{0} \dot{0} \dot{0}(\zeta, \bar{\zeta})}{r}-\frac{1}{r^{2}}\left(C_{1}-u \bar{\partial} C_{2}\right), \\
& \sigma^{0}{ }_{\mathrm{io}}=-\frac{1}{2 r^{2}}\left(C_{1}-u \bar{\partial} C_{2}\right) \text {, } \\
& \sigma^{0}{ }_{i i}=-\frac{C_{2}}{r}-\frac{1}{2 r^{2}}\left(ð C_{1}+u C_{2}\right), \\
& \sigma^{1}{ }_{i 0}=0 \text {, } \\
& \sigma^{1}{ }_{\mathrm{ii}}=0 \text {. }
\end{aligned}
$$

The equation $\check{\partial}^{2} \stackrel{(1)}{\sigma}_{0}{ }_{0 \dot{0}}=C_{2}$, which reveals an unphysical angular singularity in $\stackrel{(1)}{\sigma} 0$ io can be integrated, as in the previous section, in the two separate patches $(a)$ and $(b)$ of $S^{2}$ of section 2 , and a regular $\stackrel{(1)}{\sigma}_{0} 0$ de defined in each patch by

$$
\begin{aligned}
& \operatorname{Patch}(a): \stackrel{(1)}{\sigma}_{\dot{0} \dot{0}}{ }^{(a)}=-\frac{1}{4 \sqrt{\pi}}\left[\frac{(1+2 \zeta \bar{\zeta}) \beta_{\frac{1}{2}}-\bar{\zeta} \beta_{-\frac{1}{2}}}{\bar{\zeta}^{2}}\right] P^{-\frac{1}{2}}, \\
& \text { Patch }(b): \stackrel{(1)}{\sigma}_{\dot{0} \dot{0}}{ }^{(b)}=+\frac{1}{4 \sqrt{\pi}}\left[\zeta^{2} \beta_{\frac{1}{2}}-\zeta(2+\zeta \bar{\zeta}) \beta_{-\frac{1}{2}}\right] P^{-\frac{1}{2}},
\end{aligned}
$$

with $P=\frac{1}{2}(1+\zeta \bar{\zeta})$. The gauge transformation connecting the two solutions is given by equations (2.47) and (2.48).

An alternative and more elegant way of expressing these solutions is to give them in terms of covariant objects. We choose

$$
\stackrel{(1)}{\sigma}_{0 \dot{00}}=\frac{\left(o_{\dot{A}} V^{\dot{A}}\right)^{2}}{\iota_{\dot{A}} V^{\dot{A}}}-\frac{\mathfrak{F}}{\iota_{\dot{A}} V^{\dot{A}}\left(\iota_{\dot{A}} W^{\dot{A}}\right)^{2}},
$$

where $\zeta_{\mathbf{a}}{ }^{\dot{A}}=\left(o^{\dot{A}}, \iota^{\dot{A}}\right)$ is the zweibein or spinor dyad defined in section 2 .

The first term on the right-hand side of equation (3.11) is a particular solution of $\check{\partial}^{2} \stackrel{(1)}{\sigma}_{0 \dot{0}}{ }_{0}=C_{2}=\iota_{\dot{A}} V^{\dot{A}}$, where $V^{\dot{A}}=\left(V^{\dot{0}}, V^{\dot{\mathrm{i}}}\right)$ is a constant spinor with $V^{\dot{0}}=-\frac{\beta_{-\frac{1}{2}}}{2 \sqrt{\pi}}$ and $V^{\mathrm{i}}=\frac{\beta_{\frac{1}{2}}}{2 \sqrt{\pi}}$. The second term is the solution of the homogeneous equation $\partial^{2} \stackrel{(1)}{\sigma}_{(H)}^{0} \dot{0}_{00}=0$, 
which is used to move the singularity of $\stackrel{(1)}{\sigma}_{0}{ }_{00}$ from one point to another on the sphere $S^{2}$. Here $W^{\dot{A}}$ is an arbitrary spinor that moves the singularity about on $S^{2}$. To ensure that ${ }_{\sigma}^{(1)} 0_{\dot{0} 0}$ approaches a well defined limit as $\bar{\zeta} \rightarrow \frac{V^{\mathrm{i}}}{V^{0}}$, which is the zero of $\iota_{\dot{A}} V^{\dot{A}}$, we choose $\mathfrak{F}$ to be the value of $\left(\iota_{\dot{A}} W^{\dot{A}}\right)^{2}\left(o_{\dot{A}} V^{\dot{A}}\right)^{2}$ at $\bar{\zeta}=\frac{V^{\mathrm{i}}}{V^{0}}$. Thus

$$
\mathfrak{F}=\left.\left(\iota_{\dot{A}} W^{\dot{A}}\right)^{2}\left(o_{\dot{A}} V^{\dot{A}}\right)^{2}\right|_{\bar{\zeta}=\frac{V^{\mathrm{i}}}{V^{0}}}=\left(V_{\dot{A}} W^{\dot{A}}\right)^{2} .
$$

A useful coordinate representation of $\zeta_{\mathbf{a}}{ }^{\dot{ }}$ is given by

$$
o_{\dot{A}}=\frac{1}{\sqrt{P}}(1, \zeta) \quad \text { and } \quad \iota_{\mathrm{A}}=\frac{1}{\sqrt{\mathrm{P}}}(-\bar{\zeta}, 1) .
$$

In this coordinate system, the choice $W^{\dot{A}}=(-1,0)$ shifts the 'natural' singularity $\bar{\zeta}=\frac{V^{\text {i }}}{V^{0}}$ to the point $\bar{\zeta}=0$.

Finally, the gauge field $v_{\dot{A}}$ responsible for this shift of the singularity is given by

$$
\begin{aligned}
& v_{0}=-\frac{V_{\dot{A}} W^{\dot{A}}}{\iota_{\dot{A}} W^{\dot{A}}}-o_{\dot{A}} V^{\dot{A}} \log \left(\frac{\iota_{\mathrm{A}} \mathrm{V}^{\mathrm{A}}}{\iota_{\mathrm{A}} \mathrm{W}^{\mathrm{A}}}\right), \\
& v_{\dot{1}}=\iota_{\dot{A}} V^{\dot{A}} \log \left(\frac{\iota_{\mathrm{A}} \mathrm{V}^{\mathrm{A}}}{\iota_{\mathrm{A}} \mathrm{W}^{\mathrm{A}}}\right) .
\end{aligned}
$$

The relations between the first potential $\sigma^{C} \dot{A} \dot{B}$ and the second potential $\rho_{\dot{A}}{ }^{B C}$ can now be integrated. Using, in addition, condition (1.15) on $\rho_{\dot{A}}{ }^{B C}$, we have an exact solution in null gauge $\left(\rho_{0}{ }^{10}=0=\rho_{0}{ }^{11}\right)$ :

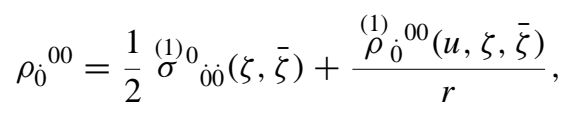

where

$$
\begin{aligned}
& \partial^{2} \stackrel{(1)}{\rho_{0}}{ }_{0}^{00}=\frac{1}{2} \partial C_{1}+\frac{u}{4} C_{2}, \\
& \rho_{0}{ }^{01}=\frac{\stackrel{(1)}{\rho_{0}}{ }_{0}^{01}(u, \zeta, \bar{\zeta})}{r},
\end{aligned}
$$

where

$$
\begin{aligned}
& \text { ð } \stackrel{(1)}{\rho}_{0}^{01}=-\frac{u}{2} C_{2}, \\
& \rho_{\mathrm{i}}^{00}=\frac{1}{4 r}\left(C_{1}-u \bar{\delta} C_{2}\right), \\
& \rho_{\mathrm{i}}{ }^{10}=-\frac{u}{2 r} C_{2}, \\
& \rho_{\mathrm{i}}{ }^{11}=0 .
\end{aligned}
$$

The singular components of $\rho_{\dot{A}}^{B C}$ are again $\stackrel{(0)}{\rho}_{0} 00$ and $\stackrel{(1)}{\rho}_{0}{ }_{0}^{00}$, where $\stackrel{(0)}{\rho}_{0}^{00}=\frac{1}{2} \stackrel{(1)}{\sigma}_{0}^{0}$ oi and where $\stackrel{(1)}{\rho}_{0} 00$ is found in a similar manner (see equation (3.11)) but with $C_{2}$ replaced by 
$\frac{1}{2} ð C_{1}+\frac{u}{4} C_{2}=o_{A} \hat{V}^{A}$ with $\hat{V}^{A}=\left(\hat{V}^{0}, \hat{V}^{1}\right)=\left(-\frac{\delta_{-\frac{1}{2}}+\frac{u}{2} \beta_{-\frac{1}{2}}}{4 \sqrt{\pi}}, \frac{\delta_{\frac{1}{2}}+\frac{u}{2} \beta_{1}}{4 \sqrt{\pi}}\right)$. The gauge field $\chi^{A}$ responsible for the shift of the singularity of the component $\stackrel{(1)}{\rho}_{0} 00$ on the sphere is given by

$$
\begin{aligned}
& \chi^{0}=-\frac{\hat{V}_{A} \hat{W}^{A}}{o_{A} \hat{W}^{A}}-\iota_{A} \hat{V}^{A} \log \left(\frac{\mathrm{o}_{\mathrm{A}} \hat{V}^{\mathrm{A}}}{\mathrm{o}_{\mathrm{A}} \hat{\mathrm{W}}^{\mathrm{A}}}\right), \\
& \chi^{1}=2 o_{A} \hat{V}^{A} \log \left(\frac{\mathrm{o}_{\mathrm{A}} \hat{\mathrm{V}}^{\mathrm{A}}}{\mathrm{o}_{\mathrm{A}} \hat{\mathrm{W}}^{\mathrm{A}}}\right),
\end{aligned}
$$

where now $\hat{W}^{A}$ is an arbitrary spinor that moves the singularity of $\stackrel{(1)}{\rho}_{0}^{00}$ from one point to another on $S^{2}$.

Note that in the two gauge fields $\chi^{A}$ and $v_{\dot{A}}$, there is a logarithm that changes by $2 \pi i$ on a circuit around the equator. The associated changes in the $\chi^{A}$ and the $v_{\dot{A}}$ have been referred to as the 'glitches' or jumps in the gauge fields. On $\mathcal{I}$ the glitches are two functions of $u, \zeta$ and $\bar{\zeta}$, namely $\iota_{A} \hat{V}^{A}$ and $\iota_{\dot{A}} V^{\dot{A}}$. Penrose observed that the vanishing of these glitches on $\mathcal{I}$ defines a curve on $\mathcal{I}$ which is identical to the so-called twistor curves of flat space, defined by the intersection of a twistor surface with $\mathcal{I}$ [12]. One attempt to understand twistors in asymptotically flat spacetime is to see if this idea of the glitches and the curve on which they vanish can be generalized to the situation where there is gravitational radiation. This problem will be discussed, but not solved, in section 5 .

\section{Rarita-Schwinger Equations in Ricci-flat spacetime}

In this section we study the solutions of the Rarita-Schwinger equations in Ricci-flat spacetime. First of all, we note that now we do not have the gauge-invariant spin- $\frac{3}{2}$ fields $\psi_{\dot{A} \dot{B} \dot{C}}$ to fall back upon. As in the flat case, we choose Bondi coordinates $(u, r, \zeta, \bar{\zeta})$, and the associated complex null tetrad or vierbein $\lambda^{\mu}{ }_{a}=\left(l^{\mu}, n^{\mu}, m^{\mu}, \bar{m}^{\mu}\right)$ may be written as [13]

$$
\begin{aligned}
l^{\mu} & =\delta_{1}^{\mu}, \\
n^{\mu} & =\delta_{0}^{\mu}+U \delta_{1}^{\mu}+X^{i} \delta_{i}^{\mu}, \\
m^{\mu} & =\omega \delta_{1}^{\mu}+\xi^{i} \delta_{i}^{\mu} .
\end{aligned}
$$

With this choice of coordinate system and tetrad, the tetrad derivatives assume the form

$$
\begin{aligned}
D & =l^{\mu} \frac{\partial}{\partial x^{\mu}}=\frac{\partial}{\partial r}, \\
\Delta & =n^{\mu} \frac{\partial}{\partial x^{\mu}}=\frac{\partial}{\partial u}+U \frac{\partial}{\partial r}+X^{\zeta} \frac{\partial}{\partial \zeta}+X^{\bar{\zeta}} \frac{\partial}{\partial \bar{\zeta}}, \\
\delta & =m^{\mu} \frac{\partial}{\partial x^{\mu}}=\omega \frac{\partial}{\partial r}+\xi^{\zeta} \frac{\partial}{\partial \zeta}+\xi^{\bar{\zeta}} \frac{\partial}{\partial \bar{\zeta}}, \\
\bar{\delta} & =\bar{m}^{\mu} \frac{\partial}{\partial x^{\mu}}=\bar{\omega} \frac{\partial}{\partial r}+\bar{\xi}^{\zeta} \frac{\partial}{\partial \zeta}+\bar{\xi}^{\bar{\zeta}} \frac{\partial}{\partial \bar{\zeta}},
\end{aligned}
$$

where $\overline{\left(\xi^{\zeta}\right)}=\bar{\xi}^{\bar{\zeta}}$ and $\overline{\left(\xi^{\bar{\zeta}}\right)}=\bar{\xi}^{\zeta}$. The complete asymptotically flat solution of the vacuum Einstein equations together with the definitions of the various quantities involved can be found in [13]. For us, the asymptotic behaviour of the shear $\sigma(u, r, \zeta, \bar{\zeta})$ of the light cones $u=$ constant is of particular importance. Specifically, $\sigma(u, r, \zeta, \bar{\zeta})=\frac{\boldsymbol{\sigma}^{0}(u, \zeta, \bar{\zeta})}{r^{2}}+\mathrm{O}\left(r^{-4}\right)$.

Before we begin working with the Rarita-Schwinger equations (1.7) and (1.8) in Ricciflat spacetime, we note that $\sigma^{1}{ }_{00}$ and $\sigma^{1}{ }_{0 i}$ can again be set equal to zero by the imposition of the null gauge condition, whereby the gauge fields $v_{0}$ and $v_{i}$ are restricted to be functions of $u, \zeta$ and $\bar{\zeta}$ alone. 
We assume the same asymptotic fall-off in $r$ of the $\sigma$ 's as that in the flat case. Thus

$$
\begin{aligned}
& \sigma_{\dot{0} \dot{0}}^{0}=\frac{\stackrel{(1)}{\sigma} \dot{0} \dot{0}}{r}+\frac{\stackrel{(2)}{\sigma} \dot{0} \dot{0}}{r^{2}}+\mathrm{O}\left(r^{-3}\right), \\
& \sigma_{0 \mathrm{i}}^{0}=\frac{\stackrel{(1)}{\sigma}_{0} \mathrm{i} \mathrm{i}}{r}+\frac{\stackrel{(2)}{\sigma}_{0} 0_{\mathrm{o}}}{r^{2}}+\mathrm{O}\left(r^{-3}\right) \text {, }
\end{aligned}
$$

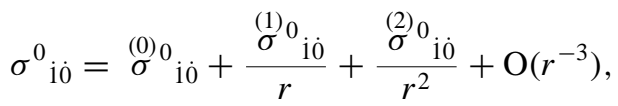

$$
\begin{aligned}
& \sigma^{0} \mathrm{ii}=\stackrel{(0)}{\sigma}_{0} \mathrm{ii}+\frac{\stackrel{(1)}{\sigma}^{0} \mathrm{ii}}{r}+\frac{\stackrel{(2)}{\sigma}_{0} \mathrm{ii}}{r^{2}}+\mathrm{O}\left(r^{-3}\right), \\
& \sigma^{1} \dot{\mathrm{i} 0}=\frac{\stackrel{(1)}{\sigma}{ }_{\mathrm{i} 0}}{r}+\frac{\stackrel{(2)}{\sigma} 1 \mathrm{i} \dot{0}}{r^{2}}+\mathrm{O}\left(r^{-3}\right) \text {, } \\
& \sigma^{1}{ }_{\mathrm{ii}}=\frac{\stackrel{(1)}{\sigma} 1 \mathrm{ii}}{r}+\frac{\stackrel{(2)}{\sigma} 1 \mathrm{ii}}{r^{2}}+\mathrm{O}\left(r^{-3}\right) \text {. }
\end{aligned}
$$

The $r$-independent terms in the $\sigma^{0}{ }_{i 0}$ and $\sigma^{0}{ }_{i i}$ series can be made to vanish by using the additional gauge freedom which further reduces $v_{0}$ and $v_{1}$ to functions of $\zeta$ and $\bar{\zeta}$ only. Besides, since ${ }_{\sigma}^{(1)} 1$ io 1 is time-independent (follows from the Rarita-Schwinger equations (1.7) and (1.8)), and since it transforms as

$$
\stackrel{(1)}{\sigma}{ }_{10} \longrightarrow \stackrel{(1)}{\sigma}{ }_{10}+v_{i}+ð v_{0} \text {, }
$$

we can set $\stackrel{(1)}{\sigma}_{1}$ i $0=0$ by defining $v_{\mathrm{i}}$ in terms of $v_{0}$ in the above relation.

Incorporating these simplifications into the tetrad version of equations (1.7) and (1.8) and then comparing the coefficients of the different powers of $\frac{1}{r}$, we obtain

$$
\begin{aligned}
& \frac{\partial \stackrel{(1)}{\sigma} 0 \dot{0} \dot{0}}{\partial u}=0=\frac{\partial \stackrel{(1)}{\sigma} 0 \dot{0} \mathrm{i}}{\partial u}, \\
& \stackrel{(1)}{\sigma}^{0} \dot{10}=0=\stackrel{(2)}{\sigma}_{0} \dot{00} \text {, } \\
& \stackrel{(2)}{\sigma}_{1}{ }_{10}=\sigma^{0} \stackrel{(1)}{\sigma}_{0}{ }_{00} \text {, } \\
& \stackrel{(1)}{\sigma}_{0} \dot{0}+\partial{ }^{(1)}{ }_{0} \dot{0}=0 \text {, } \\
& \stackrel{(2)}{\sigma}_{1}{ }_{\mathrm{ii}}=\boldsymbol{\sigma}^{0} \stackrel{(1)}{\sigma}_{0}{ }_{\mathrm{o}} \text {, } \\
& -\stackrel{(2)}{\sigma} 00 \\
& \stackrel{(2)}{\sigma}_{i}{ }_{\mathrm{ii}}=-\frac{1}{2} \partial\left(\overline{\boldsymbol{\sigma}}^{0} \stackrel{(1)}{\sigma}_{\mathrm{ii}}\right)+\frac{1}{2} \partial \stackrel{(2)}{\sigma}_{0 \mathrm{i}} \text {, }
\end{aligned}
$$

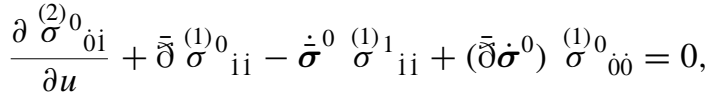

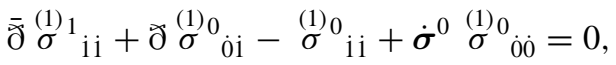

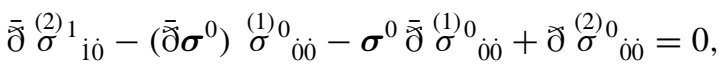

where the dot on the shear signifies the derivative with respect to the retarded time $u$.

Some of the above equations are reminiscent of equations (2.36)-(2.43) in the flat case. This similarity will be our guide (section 5) in the search for conserved quantities similar to $C_{1}$ and $C_{2}$ of the flat space solution. In the next section, we shall, in fact, discuss the issue as to whether it is at all possible to obtain conserved quantities like $C_{1}$ and $C_{2}$ by solving 
the Rarita-Schwinger equations in Ricci-flat spacetimes. We note that the asymptotic shear $\sigma^{0}(u, \zeta, \bar{\zeta})$ of the vacuum spacetime couples directly to the leading terms of the RaritaSchwinger fields. As we shall see in section 5, this coupling acts as the main impediment to defining a conserved charge.

We now proceed to expressing the above solution in terms of the components of the potential $\rho_{\dot{A}}{ }^{B C}$ by making use of the tetrad versions of equations (1.13) and (1.14). The solution again simplifies by choosing the null gauge in which $\rho_{0}{ }^{10}=0=\rho_{0}{ }^{11}$. As before, we assume that the tetrad components of $\rho_{\dot{A}}{ }^{B C}$ have the same asymptotic fall-off in $r$ as that in the flat case. Symbolically, we write

$$
\rho=\stackrel{(0)}{\rho}+\frac{\stackrel{(1)}{\rho}}{r}+\frac{\stackrel{(2)}{\rho}}{r^{2}}+\mathrm{O}\left(r^{-3}\right)
$$

where $\rho$ represents all the non-zero components of $\rho_{\dot{A}}{ }^{B C}$ in the tetrad basis.

By the residual gauge freedom, $\stackrel{(0)}{\rho}{ }_{i} 00$ and $\stackrel{(0)}{\rho}_{i} 01$ are set equal to zero, which leaves the gauge fields $\chi^{0}$ and $\chi^{1}$ as functions of $\zeta$ and $\bar{\zeta}$ alone. We then have the following information:

$$
\begin{aligned}
& \stackrel{(0)}{\rho}{ }_{0} 01=\stackrel{(0)}{\rho}{ }_{\mathrm{i}}{ }^{10}=\stackrel{(0)}{\rho}{ }_{\mathrm{i}}{ }^{11}=\stackrel{(1)}{\rho}{ }_{\mathrm{i}}{ }^{01}=0, \\
& \frac{1}{2} \stackrel{(1)}{\sigma} 0 \\
& -\frac{u}{2} \partial \stackrel{(1)}{\sigma} 0 \dot{0} \dot{0}=\stackrel{(1)}{\rho}{ }_{0}^{01}, \\
& -\frac{1}{2} \stackrel{(1)}{\sigma}_{0}{ }_{0} \mathrm{i}=\partial \stackrel{(0)}{\rho}{ }_{0}{ }_{0} 00, \\
& \frac{1}{2} \stackrel{(2)}{\sigma}{ }_{i 0}=\sigma^{0} \stackrel{(0)}{\rho}_{\dot{0}}{ }^{00}, \\
& \frac{1}{2} \stackrel{(1)}{\sigma}_{\mathrm{i}} \mathrm{ii}=\frac{\partial \stackrel{(1)}{\rho}{ }_{\mathrm{i}}{ }^{10}}{\partial u}-\dot{\boldsymbol{\sigma}}^{0} \stackrel{(0)}{\rho_{0}}{ }_{0}^{00}, \\
& \frac{1}{2} \stackrel{(1)}{\sigma}_{1 i}=\frac{\partial \stackrel{(1)}{\rho}{ }_{i} 11}{\partial u} \text {. }
\end{aligned}
$$

Equations (4.24) and (4.25) can be immediately integrated to give

$$
\stackrel{(1)}{\rho}{ }_{\mathrm{i}}^{11}=\frac{1}{2} \int_{-\infty}^{u} \mathrm{~d} u^{\prime} \stackrel{(1)}{\sigma}{ }^{1} \mathrm{ii}
$$

and

$$
\stackrel{(1)}{\rho}{ }_{\mathrm{i}}{ }^{10}=\frac{1}{2} \int_{-\infty}^{u} \mathrm{~d} u^{\prime} \stackrel{(1)}{\sigma}_{\mathrm{ii}}+\frac{\boldsymbol{\sigma}^{0}}{2} \stackrel{(1)}{\sigma}_{0}^{0} \dot{0},
$$

where, as in the flat case, we have set the time-independent functions of integration in both $\stackrel{(1)}{\rho} \mathrm{i}_{\mathrm{i}}{ }^{11}$ and $\stackrel{(1)}{\rho}_{\mathrm{i}}{ }^{10}$ equal to zero by making use of the residual gauge freedom in the $\chi$ 's.

To proceed further, we make the assumption $\stackrel{(2)}{\rho}_{\mathrm{i}}{ }^{10}=0$. In flat space, equations (1.13)(1.15) ensured the vanishing of the component $\stackrel{\left(_{\rho}^{2}\right)}{{ }_{1}} 1^{10}$. Although equation (1.15) does not have a realization in curved space, the imposition of the very mild condition $\stackrel{(2)}{\rho}{ }_{\mathrm{i}} 10=0$ on the curved space $\rho$ 's considerably improves the situation by making the equations for $\stackrel{(1)}{\rho}_{0}{ }^{00}$ and $\stackrel{(1)}{\rho}_{i} 00$ separable and thereby removing any arbitrariness in the solution of $\rho_{\dot{A}}{ }^{B C}$. Furthermore, the condition does not lead to any ambiguity in the solution.

Finally, we remark that although the equations for $\stackrel{(1)}{\rho}_{0}^{00}$ and $\stackrel{(1)}{\rho}_{i}{ }^{00}$ can, in principle, be solved in terms of the $\sigma$ 's and the shear, in actual practice they are extremely complicated 
and difficult to integrate in closed form. It suffices to comment that $\stackrel{(1)}{\rho}{ }_{0}^{00}$ becomes badly singular in curved space.

As the equations for the $\sigma$ and the $\rho$ are quite similar in structure to their flat space counterparts, we shall, in the next section, take advantage of this fact to try to construct twistor charges in our Ricci-flat spacetime.

\section{Charges in an arbitrary vacuum spacetime}

Using the results of the previous section, we now elucidate the general problem of defining conserved charges for massless spin- $\frac{3}{2}$ fields. Before considering the question as to whether it is at all possible to define conserved charges in an arbitrary vacuum spacetime, it is worthwhile to reconsider the concept of charges in a Minkowski space by viewing them solely as conserved $(u$-independent) quantities appearing in certain components of the potential $\sigma^{C} \dot{A} \dot{B}$.

We begin by trying to see what information we can extract about any conserved quantities in a Minkowski space by restricting ourselves to solutions of the Rarita-Schwinger equations alone without using the fields $\psi_{\dot{A} \dot{B} \dot{C}}$. The following relations are obtained by considering asymptotic solutions of the Rarita-Schwinger equations in the null tetrad basis:

$$
\begin{aligned}
& \frac{\partial \stackrel{(1)}{\sigma}_{0} \dot{0} \dot{0}}{\partial u}=0=\frac{\partial \stackrel{(1)}{\sigma} 0 \dot{0} \mathrm{i}}{\partial u}, \\
& \text { ठ } \stackrel{(1)}{\sigma}_{0}^{0} \dot{0}=-\stackrel{(1)}{\sigma}_{0}{ }_{0} \mathrm{i}, \\
& \stackrel{(1)}{\sigma}{ }_{\mathrm{ii}}=\partial \stackrel{(1)}{\sigma}_{0}{ }_{\mathrm{oi}}+\bar{\partial}{ }_{\sigma}^{(1)}{ }_{\mathrm{ii}} \text {. }
\end{aligned}
$$

Combining equations (6.2) and (6.3), we obtain

$$
\partial^{2} \stackrel{(1)}{\sigma}_{0 \dot{0}}=\bar{\partial} \stackrel{(1)}{\sigma}_{1}{ }_{i i}-\stackrel{(1)}{\sigma}_{0}{ }_{i i} \text {. }
$$

Because $\stackrel{(1)}{\sigma} 0_{00}$ is a time-independent (equation (5.1)), the quantity on the right-hand side of equation (5.4) must be time-independent. Now, since the spin weights of $\stackrel{(1)}{\sigma} 0_{00},{ }_{\sigma}^{(1)}{ }_{i 1}$, and $\overline{\mathrm{\partial}} \stackrel{(1)}{\sigma}^{1}{ }_{\mathrm{ii}}$ are $-\frac{3}{2}, \frac{1}{2}$, and $\frac{1}{2}$ respectively, we see immediately that a time-independent $l=\frac{1}{2}$ component can be generated from the quantity on the right-hand side of equation (5.4) which makes $\stackrel{(1)}{\sigma} 0_{\dot{0} 0}$ a singular function on the sphere. This object has the right character of a conserved charge and is in fact the $C_{2}$ of section 2.

We now try to emulate this procedure to generate a free time-independent object as a conserved charge by considering the Rarita-Schwinger equations in an arbitrary Ricci-flat spacetime. From equations (4.11) and (4.16), we obtain the following equation which is analogous to equation (6.4) considered above for the flat case:

$$
\partial^{2} \stackrel{(1)}{\sigma}_{0}^{0} \dot{0}=\bar{\partial} \stackrel{(1)}{\sigma}{ }_{i i}-\stackrel{(1)}{\sigma}^{0}{ }_{i i}+\dot{\sigma}^{0} \stackrel{(1)}{\sigma}_{0} 0 \dot{0} \text {. }
$$

Since $\stackrel{(1)}{\sigma}_{00}$ is time-independent (equation (4.8)), the right-hand side of the above equation must also be time-independent. We then have

$$
\overline{\bar{\partial}} \stackrel{(1)}{\sigma}{ }_{i i}-\stackrel{(1)}{\sigma}_{i i}+\dot{\sigma}^{0} \stackrel{(1)}{\sigma}_{0 \dot{0}}=C_{2}(\zeta, \bar{\zeta}) .
$$

Since the spin weights of the terms $\stackrel{(1)}{\sigma}_{0}{ }_{i \mathrm{i}}$ and $\dot{\boldsymbol{\sigma}}^{0} \stackrel{(1)}{\sigma}_{0}{ }_{\mathrm{o} \dot{0}}$ are both $\frac{1}{2}$, there is the possibility of generating a time-independent $l=\frac{1}{2}$ component on the right-hand side of equation (5.6). $C_{2}(\zeta, \bar{\zeta})$ stands for this particular component. All time-independent higher harmonics 
$\left(l=\frac{3}{2}, \frac{5}{2}, \ldots\right)$ can be disposed of by choosing suitable initial conditions for the terms on the left-hand side of equation (5.6).

Equation (5.5) now gives

$$
\partial^{2} \stackrel{(1)}{\sigma}{ }_{0 \dot{0}}=C_{2} \text {. }
$$

As in the flat case, $\stackrel{(1)}{\sigma} 0_{00}$ is singular on the sphere $S^{2}$.

$C_{2}$ is indeed the same conserved quantity as that encountered in the flat case in section 2 constituting one component of the twistor charge $Q$. Let us now see if we can also find the other component $C_{1}$ of the charge. If this $C_{1}$ is to be a conserved quantity, it should appear, as in the flat case, as a time-independent function of integration in the component $\stackrel{(2)}{\sigma}_{0 \mathrm{i}}$. To find it, we first note that equation (5.6) allows us to define ${ }_{\sigma}^{(1)}{ }_{0} \mathrm{ii}$ in terms of $C_{2}(\zeta, \bar{\zeta})$, the arbitrarily specified 'gravitational news' $\boldsymbol{\sigma}^{0}$, and the free spin- $\frac{3}{2}$ 'radiation' term $\stackrel{(1)}{\sigma}{ }_{\mathrm{ii}}$. Thus,

$$
\stackrel{(1)}{\sigma}_{\mathrm{ii}}=-C_{2}(\zeta, \bar{\zeta})+\overline{\bar{\partial}} \stackrel{(1)}{\sigma}{ }_{\mathrm{ii}}+\dot{\boldsymbol{\sigma}}^{0} \stackrel{(1)}{\sigma}{ }_{00}{ }_{0} .
$$

We now substitute $\stackrel{(1)}{\sigma}_{\text {ii }}$ from equation (5.8) into equation (4.15), which can then be integrated to yield

$$
\begin{aligned}
& \stackrel{(2)}{\sigma}_{0}{ }_{0 \mathrm{i}}=-C_{1}(\zeta, \bar{\zeta})+u \bar{\partial} C_{2}(\zeta, \bar{\zeta})-\bar{\partial}\left(\sigma^{0} \stackrel{(1)}{\sigma}_{0}{ }_{0}\right) \\
& -\left(\overline{\widetilde{\partial}} \sigma^{0}\right) \stackrel{(1)}{\sigma}_{\dot{0} \dot{0}}-\int_{-\infty}^{u} \mathrm{~d} u^{\prime}\left(\overline{\widetilde{\partial}}^{2}-\dot{\bar{\sigma}}^{0}\right) \stackrel{(1)}{\sigma}^{1} \mathrm{ii} .
\end{aligned}
$$

$C_{1}(\zeta, \bar{\zeta})$, being again the $l=\frac{1}{2}$ harmonic of the form seen earlier in equation (2.43) of flat space, has the right appearance of the other component of the twistor charge, our desideratum. Unfortunately $C_{1}$ cannot now be identified with certainty as a conserved quantity similar to that obtained in the flat case, since it is not clear that the time-dependent singular terms (the third and the fourth terms on the right-hand side of equation (5.9)) do not contribute a time-dependent $l=\frac{1}{2}$ component to be added to $C_{1}$. However, if we assume that $C_{1}$ is, in fact, conserved (note that $C_{2}$ is always conserved), we are still presented with the problem of showing the existence of the 'glitches' and the associated curves on $\mathcal{I}$, defined by the vanishing of the glitches (discussed in section 3 in the context of flat space), as well as finding and investigating the properties of these curves. The conjecture is that the space of these curves is indeed the generalization of twistor space to asymptotically flat vacuum spacetimes and that this generalized twistor space 'encodes' the information of the spacetime itself.

\section{Conclusion}

We briefly summarize here what we have accomplished in this work. First, the asymptotic retarded solutions of the massless spin- $\frac{3}{2}$ fields in Minkowski space have been found. The solutions reveal the existence of four complex conserved quantities in the fields $\psi_{\dot{A} \dot{B} \dot{C}}$. The space of these four complex quantities can be shown to be isomorphic to flat twistor space. With appropriate choices of gauge, the equations for the first and second potentials can be integrated asymptotically. The conserved quantities reappear in certain singular behaviour of the potentials, the singularities being of the Dirac string type, movable by gauge transformations. An exact special solution, the 'quasi-Coulomb' solution-the analogue of the Coulomb solution of the Maxwell equations-was found and analysed, which exactly exhibits the conserved quantities. 
These calculations have then been repeated for the case of asymptotically flat vacuum spaces. Once again one can see where quantities similar to the four complex conserved charges arise.

Unfortunately, it still remains an unsolved problem as to how a curved twistor space could be constructed from these quantities. It is clear, however, that the obstruction to the flat space twistor construction is the existence of gravitational radiation, in the form of Bondi shear.

\section{Acknowledgments}

The authors wish to acknowledge and thank Professor Roger Penrose and Lionel Mason for valuable discussions and comments. They would also like to thank an unknown referee for suggesting that there might be a connection between the twistor charges considered in this work and the 'supercharge' studied in supergravity theories [14].

\section{References}

[1] Buchdahl H A 1958 Nuovo Cim. 1096

[2] Penrose R 1992 Gravitation and Modern Cosmology ed A Zichichi, N de Sabbata, and N Sánchez (New York: Plenum Press)

[3] Penrose R 1990 Twist. Newslett. 316

[4] Dirac P A M 1936 Proc. R. Soc. A 155447

[5] Rarita W and Schwinger J 1941 Phys. Rev. 6061

[6] Chinea F J 1984 Phys. Rev. Lett. 52322

[7] Deser S and Zumino B 1976 Phys. Lett. 62B 3335

[8] Freedman D Z, van Nieuwenhuizen P and Ferrara S 1976 Phys. Rev. D 133214

Freedman D Z and van Nieuwenhuizen P 1976 Phys. Rev. D 14912

[9] Janis A I and Newman E T 1965 J. Math. Phys. 6902

[10] Newman E T and Penrose R 1968 Proc. R. Soc. A 305175

[11] Penrose R and Rindler W 1984 Spinors and Spacetime vol 1 (Cambridge: Cambridge University Press)

[12] Hansen R O and Newman E T 1975 Gen. Rel. Grav. 6361

[13] Newman E T and Tod K P 1980 General Relativity and Gravitation vol 2, ed A Held (New York: Plenum)

[14] Teitelboim C 1977 Phys. Lett. 69B 240 